\title{
COMUNICACIÓN
}

\section{Examen paleoparasitológico de sedimentos de un sitio arqueológico, Río Mayo, Chubut, Argentina}

\author{
MARTÍN H. FUGASSA*
}

\section{PALEOPARASITOLOGICAL ANALYSIS OF SEDIMENTS OF AN ARCHAEOLOGICAL SITE, RÍO MAYO, CHUBUT, ARGENTINA}

Sediments belonging to an archaeological rescue were examined for parasites. The archaeological site consists in a rockshelter, close to Río Mayo locality, southwest of Chubut province and associated to european contact, probably s. XIX. Organic remains proceeding from the pelvic girdle were examined. By means of quantitative and qualitative techniques, eggs of anoplocephalids and nematodes, Trichuris sp., were observed. Macroscopic inspection of the sample showed rodent coprolites; the paleoparasitologic analysis exhibited eggs of anoplocephalids, Trichuris sp, Capillaria sp and an unidentified ascaridid. Probable contamination of the deposit with rodent parasites is discussed.

Key words: Archaeological sediment, Coprolites, Patagonia, European contact.

\section{INTRODUCCIÓN}

El presente trabajo pretende ampliar el estudio paleoparasitológico sobre sedimentos asociados a restos humanos y vinculados al período histórico. Se procura que el aumento en el número de muestras analizadas permita gradualmente construir un panorama paleoepidemiológico del proceso de contacto europeo-aborigen en Patagonia. Asimismo, los estudios paleoparasitológicos deben simultáneamente adaptar y mejorar los métodos empleados para su aplicación a las condiciones particulares de la región ${ }^{1}$. Por tal motivo, se comparan los resultados obtenidos por medios cualitativos con aquellos logrados por técnicas cuantitativas modificadas.

Los estudios paleoparasitológicos en la arqueología de Patagonia han sido escasos ${ }^{2,3}$.
Recientes investigaciones han ampliado dichos trabajos y una parte importante de sus resultados se obtuvieron mediante el estudio de coprolitos de fauna encontrados en los sitios arqueológicos ${ }^{4-}$ ${ }^{6}$. Las muestras de origen humano siguen siendo escasas y la disponibilidad de aquellas vinculadas específicamente con el período histórico es aun más limitada por lo que este trabajo tiene entonces un doble interés.

\section{MATERIAL Y MÉTODOS}

El sitio Alero Mazquiarán es un abrigo rocoso ubicado en el sudoeste de la provincia de Chubut, Argentina, donde se halló un enterratorio múltiple compuesto por 5 individuos cuyo estudio está aun en proceso ${ }^{7}$. Se analizaron sedimentos pertenecientes a uno de los cuerpos, correspondiente a un adulto joven, de sexo masculino (Bernal

\footnotetext{
Lab. de Zoonosis Parasitarias, Depto. de Biología, Univ. Nacional de Mar del Plata - Becario CONICET. E-mail: mfugassa@mdp.edu.ar
} 
com. pers., 2006). El esqueleto se encontraba articulado, con restos tisulares y cabellos y envuelto en una tela tejida con lana de oveja. El fechado de esta prenda arrojó una antigüedad de $212 \pm 35$ años ${ }^{14}$ C A. P. (antes del presente) $)^{7}$.

Los sedimentos examinados provinieron del interior de la cintura pélvica. Se fraccionaron los sedimentos recibidos, tomándose al azar 10 muestras de $5 \mathrm{~g}$ cada una que fueron rehidratadas en $10 \mathrm{ml}$ de fosfato trisódico acuoso $0,5 \%$ (TSP) ${ }^{8}$. Posteriormente, se aplicó la técnica de Stoll modificada $^{9,10}$, consistente en la preparación de diluciones de sedimento en TSP y la posterior obtención de alícuotas de $50 \mu \mathrm{l}$ para ser observados al microscopio óptico. El conteo del número de huevos por preparado es referido a huevos por gramo, ya que se conoce el peso de la muestra usada y el volumen de solución empleado.

Para optimizar la recuperación de restos parasitarios y comparar su sensibilidad con la de la técnica cuantitativa, fue aplicada la técnica de flotación en solución saturada de sacarosa o solución de Sheater ${ }^{11}$. Para ello, las muestras fueron centrifugadas a $1.500 \mathrm{rpm}$ durante 3 minutos y luego se descartó el sobrenadante. El sedimento se resuspendió en la solución saturada de sacarosa con $10 \%$ de formol. Se examinó al microscopio óptico el material que flotó en la superficie de la solución después de 60, 120 y 180 minutos.
Debido a que se hallaron heces de roedor dentro del sedimento, éstas se separaron, fueron descriptas y luego se rehidrataron con TSP y se procesaron por sedimentación espontánea ${ }^{12}$.

\section{RESULTADOS}

El análisis paleoparasitológico cuantitativo del sedimento sólo arrojó la probable presencia de 1 huevo de tenia de $30 \mu \mathrm{m}$ de diámetro (Figura 1A).

Mediante la técnica de flotación se identificaron huevos de parásitos, aunque en muy reducido número. Se reconoció 1 huevo de Trichuris sp., 61,75 x 28,75 $\mu \mathrm{m}$ y 2 huevos de anoplocefálidos, de forma triangular, con embrióforo y ganchos visibles, con dimensiones 57,5-60,0 $\mu \mathrm{m}$ de diámetro (Figura 1B).

El examen macroscópico del sedimento produjo numerosas fecas de roedor, identificándose dos tipos: $\mathrm{X}-10$ con un textura liviana y fibrosa, de color gris verdoso y X-11 de textura compacta y lisa, de color pardo gris. Ambas con dimensiones similares.

El análisis paleoparasitológico de las heces de roedor permitió confirmar que existían 2 tipos, pertenecientes a 2 animales diferentes. Los coprolitos X-10 contenían mayormente tejido vegetal, algunos ácaros y huevos de cestodes anoplocefálidos que midieron 55,0-60,0 (57,5 \pm $2,04 ; \mathrm{n}=4) \times 57,5-61,25(59,69 \pm 1,57 ; \mathrm{n}=4) \mu \mathrm{m}$.

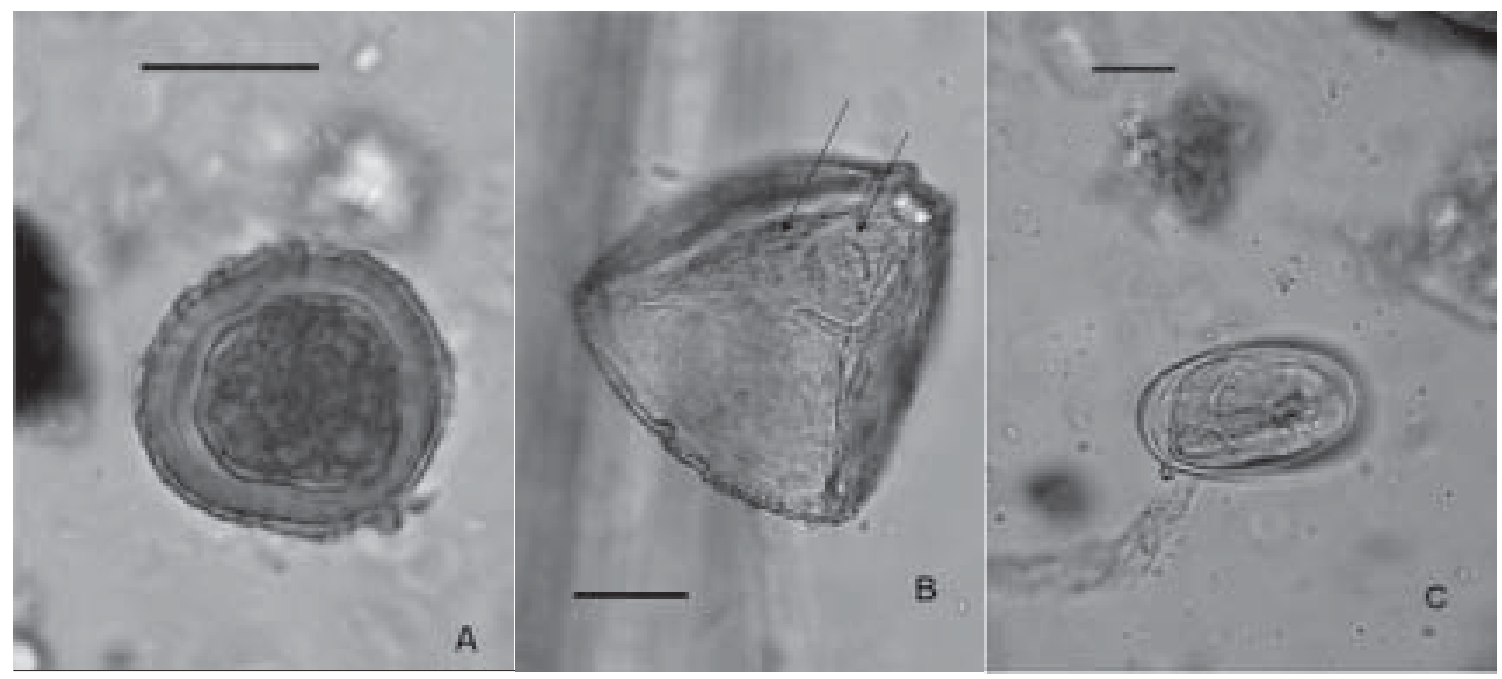

Figura 1. (A) cuerpo compatible con un huevo de tenia. (B) huevo de anoplocefálido (la flechas indican el embrióforo y los ganchos). (C) huevo de ascaridido no identificado. Barra $=20 \mu € \mathrm{~m}$. 
El contenido de los coprolitos X-11 fue menor en tejido vegetal que el de X-10. Estos presentaron huevos de pared delgada $(n=2)$, translúcida y larvados (Figura 1C) y que midieron $35,0 \times 53,0 \mu \mathrm{m}$ probablemente pertenecientes a un ascaridido; se registró 1 huevo de Capillaria sp. de 65,0 x 35,0 $\mu \mathrm{m}$ y 1 huevo de Trichuris sp. que midió $66,25 \times 52,5 \mu \mathrm{m}$.

\section{DISCUSIÓN}

La presencia de fecas de roedor en el depósito demuestra la importancia del examen macroscópico de los sedimentos antes de realizar los estudios paleoparasitológicos. Similar conclusión se logró en el análisis de los sedimentos del sitio Orejas de Burro $1^{13,14}$ donde la cavidad abdominal de un individuo fue utilizada como refugio por un roedor. Domeyko ${ }^{7}$ relató a mediados del s. XIX que los aborígenes de la región colocaban ofrendas durante la inhumación de los muertos y que incluían diversos alimentos. Por esta razón, las ofrendas colocadas junto a los cadáveres pudieron atraer a roedores. Aparentemente, los restos humanos resultan abrigos adecuados para algunas especies de roedores y ello demanda atención en la ejecución de los estudios paleoparasitológicos de depósitos arqueológicos de este tipo.

El examen paleoparasitológico de los coprolitos de roedor sugiere que los huevos de anoplocefálidos y Trichuris sp hallados en el contenido abdominal del esqueleto provienen de la contaminación con las heces de uno de los roedores presentes.

Metodológicamente, resultó útil la aplicación de una técnica alternativa ante los resultados negativos del método cuantitativo. Respecto de la aplicación del método de flotación, se realizaron 3 observaciones seriadas debido a que la solución de sacarosa es viscosa y la flotación de los huevos y quistes puede ser lenta. Sin embargo, los restos parasitarios sólo se registraron en la primera observación.

La interpretación de los resultados negativos de la técnica cuantitativa es que esta puede fallar a muy bajas densidades de huevos -un total de sólo 3 huevos fueron recuperados tras la aplicación de la técnica cualitativa. Sin embargo, la técnica cuantitativa utilizada aquí fue efectiva en otras condiciones de baja densidad de huevos ${ }^{9}$. Probablemente, estas diferencias de sensibilidad se deban a que en el sitio Alero Mazquiarán la cantidad de tejidos vegetales y otros restos orgánicos fue elevada y ello pudo retener elementos parasitarios que sí quedaron libres durante la exposición a la solución de flotación. En todos los casos se registraron pocos huevos para cada especie de helminto. Los huevos de anoplocefálidos presentaron aparato piriforme definido y una morfometría compatible con la de Moniezia sp. ${ }^{15,16}$ aunque los roedores no han sido reportados aún como hospedadores para este género $^{17,18}$. La probable presencia de 1 huevo de tenia en el sedimento abdominal no puede ser sostenida con tan poca evidencia. Asimismo, el registro de Capillaria sp, Trichuris sp y un ascadidido en las heces de roedor y a través de 1 ó 2 huevos en cada caso tampoco permite un diagnóstico específico.

Los estudios sobre Alero Mazquiarán corresponden a tiempos históricos. El interés sobre este sitio se vinculó inicialmente a los posibles resultados en función del estudio del contacto europeo-aborigen en Patagonia. Sin embargo, no se obtuvieron resultados asociados directamente con el parasitismo en humanos ya que los reportes presentados corresponderían a los de roedores.

$\mathrm{Si}$ bien los resultados paleoparasitológicos vinculados al individuo humano pueden considerarse escasos, los estudios realizados permitieron ampliar el conocimiento sobre la distribución de especies o géneros de parásitos a través del tiempo; específicamente, los efectos del contacto europeo-aborigen sobre la biogeografía de los parásitos. Asimismo, han provisto de nuevas pautas para la investigación paleoparasitológica de depósitos arqueológicos, tales como la aplicación secuencial de técnicas cuantitativas y cualitativas y la importancia de la inspección macroscópica de los sedimentos analizados.

\section{RESUMEN}

En este trabajo, que tiene como objetivo el estudio paleoparasitológico, se analizan sedimentos procedentes del rescate arqueológico de un entierro múltiple en un alero rocoso, cercano a la localidad de Río Mayo, al sudoeste del Chubut. Se obtuvieron sedimentos extraídos de la región pélvica de un esqueleto humano procedente de Patagonia y vinculado al período de contacto europeo-aborigen. Mediante técnicas 
cuantitativas y cualitativas se registraron huevos de anoplocefálidos y de Trichuris sp. La inspección macroscópica previa de la muestra evidenció fecas de roedor y su examen paleoparasitológico evidenció huevos de anoplocefálidos, Trichuris sp, Capillaria sp y un ascadidido no identificado. Por lo tanto, se advierte la posible contaminación del depósito con parásitos de roedores.

\section{REFERENCIAS}

1.- FUGASSA M H, GUICHÓN R A, DENEGRI G M, et al. Paleoparasitología en Patagonia Austral. Actas XV Congreso Nacional de Arqueología Argentina. 2004.

2.- ZIMMERMAN M R, MORILA R E. Enterobiasis in pre-Columbian America. Paleopathology Newsletter 1983; 42: 8.

3.- GONÇALVES M L, ARAÚJO A, FERREIRA L F. Human intestinal parasites in the past: new finding and a review. Memorias do Instituto Oswaldo Cruz 2003; 98 (Suppl. I): 103-18.

4.- FUGASSA M H, GUICHÓN R A. Análisis paleoparasitológico de coprolitos hallados en sitios arqueológicos de Patagonia Austral: definiciones y perspectivas. Magallania 2005; 33: 13-9.

5.- FUGASSA M H, DENEGRI G M, SARDELLA $N$ H, et al. Paleoparasitological Records in Canid Coprolite From Patagonia, Argentina. J Parasitol 2006 (en prensa).

6.- FUGASSA M H, DENEGRI G M, SARDELLA, $\mathrm{N} H$, et al. First paleoparasitological record of anoplocephalid cestodes in Patagonia Austral. Paleopathology Newsletter 2005; 131(Suppl.): 20.

7.- PÉREZ DE MICOU C. Textiles arqueológicos de la Patagonia Argentina. Hallazgos en el sitio Alero Mazquiarán, Río Mayo, Chubut. 2006. (en prensa).

8.- CALLEN E O, CAMERON T W M. A prehistoric diet revealed in coprolites. New Scientist 1960; 8: 35-40.

9.- FUGASSA M H, ARAÚJO A, GUICHÓN R A. Quantitative paleoparasitology applied to archaeological sediments. Memorias do Instituto Oswaldo
Cruz 2006; (en prensa).

10.- FUGASSA M H. Métodos de examen paleoparasitológicos en sedimentos arqueológicos. Parasitol Latinoam 2005; 60: 328.

11.- BENBROOK E A, SLOSS M W. Parasitología Clínica Veterinaria. Ed. Continental. México. 1965.

12.- LUTZ A. Schistosoma mansoni e a schistosomatose segundo observacoes feitas no brasil. Memorias do Instituto Oswaldo Cruz 1919; 11: 121-55.

13.- FUGASSA M H, BARBERENA R. Cuevas y zoonosis antiguas: paleoparasitología del sitio Orejas de Burro 1 (Santa Cruz, Argentina). Magallania 2006; remitido.

14.- FUGASSA M H. Primeros hallazgos paleoparasitológicos en sedimentos del sitio arqueológico Orejas de Burro, provincia de Santa Cruz, Argentina. Revista Argentina de Antropología Biológica 2005; 7: 116.

15.- THIENPONT D, ROCHETTE F, VANPARIJS O F J. Diagnóstico de las helmintiosis por medio del examen coproparasitológico. Janssen Research Foundation. Bélgica. 1979.

16.- BENBROOK E A, SLOSS M W. Parasitología clínica veterinaria. Cía. Editorial Continental. México. 1965.

17.- BAER J G. Monographie des cestodes de la familla des Anoplocephalidae. Bulletin Biologique de France et de Belgique (supplément X) 1927.

18.- SOULSBY E J. Parasitología y enfermedades parasitarias en los animales domésticos. Nueva Editorial Interamericana. México DF. 1987.

Agradecimientos. Agradezco al Dr. R. A. Guichón (CONICET) por facilitar el acceso a las muestras examinadas. A los Dres. Norma Sardella y Guillermo Denegri por la lectura del manuscrito. La investigación arqueológica en la zona de Río Mayo se realiza por un convenio entre el CONICET y la Secretaría de Cultura de Chubut y con apoyo de los Proyectos Antorchas 14116, PICT-SECyT 04-11759 y PIP-CONICET 02466-04. Agradezco a sus directoras, Dras. A. M. Aguerre, S. L. Burry y C. B. Pérez de Micou, responsables del rescate en el sitio y que se interesaron en obtener los sedimentos. A la Lic. Valeria Bernal, especialista en Bioarqueología, por su gentileza en las informaciones transmitidas. El trabajo de laboratorio se realizó en la Universidad Nacional de Mar del Plata con el apoyo del proyecto: PICT 03- 4-13889. 